Results Gastroenterology national training numbers have gradually increased from 673 in 2012 to 734 in 2020. Of those who began gastroenterology training in 2012, 24\% now remain in training, 65\% have completed their CCT and 5\% have left training.

$14 \%$ of gastroenterology HSTs reported being bullied or harassed at work. Gastroenterology ranked 6th among medical specialities on this issue. Although HSTs were less likely to experience bullying or harassment than consultants, there were higher rates reported by Black, Asian and minority ethnic (BAME) than white HSTs (17\% versus 11\%). BAME HSTs were also more likely to have experienced discrimination than white HSTs (9\% versus 7\%), and women HSTs were three times as likely to experience discrimination as men $(15 \%$ versus $4 \%)$.

Gastroenterology HSTs ranked 5th most at risk of burnout among medical specialities, with rates of moderate and high burnout risk of $39 \%$ and $12 \%$ respectively. Male HSTs reported slightly greater risks of burnout than female HSTs and BAME HSTs reported markedly higher rates of high burnout risk than white HSTs (15\% versus 9\%). 62\% of HSTs said that work had impacted on their relationship with their partner and $58 \%$ with their children. $33 \%$ of trainees reported a deterioration in morale since the previous year and only $20 \%$ an improvement.

$82 \%$ of HSTs said they found their specialty work satisfying always or often, but only $22 \%$ their general internal medicine work. A smaller proportion of gastroenterology HSTs work less than full time (LTFT) than in other medical specialities (11\% versus 17\%). When asked if they wanted to train LTFT if given the opportunity, a surprising $46 \%$ of gastroenterology HSTs said they would.

Conclusions There were huge challenges facing gastroenterology HSTs before COVID-19. Worrying trends in bullying, discrimination, morale and burnout need to be addressed urgently. As time for training shortens, we must improve trainee experiences and the quality of training to protect the future gastroenterology workforce.

\section{PTU-84 ACCELERATED ACCREDITATION FOR BOWEL CANCER SCREENING COLONOSCOPIST IN CHESHIRE AND MERSEYSIDE}

${ }^{1}$ Karen Llyod, ${ }^{1}$ Carys Kinsella, ${ }^{2}$ Neil Haslam, ${ }^{3}$ Asish Bassi. ${ }^{1}$ Chesire and Merseyside Cancer Alliance, Liverpool, UK; ${ }^{2}$ Liverpool University Foundation Trust, Liverpool, UK; ${ }^{3}$ St Helens And Knowsley Nhs Trust, Prescot, UK

10.1136/gutjpl-2021-BSG.384

Introduction In 2020, as the COVID pandemic spread through the UK, Bowel Cancer screening invitations were paused. In Cheshire and Merseyside (C\& $\&$ ) there were as many as 100,000 patients waiting for their invitations. In addition, there were a large number of patients who had already been tested but waiting for colonoscopy after their positive FIT test. It is also expected that there will be an age extension to include FIT test for those aged over 50 years as the bowel scope programme is ceased. A workforce analysis suggested that there was a significant gap in the number of endoscopists needed to deliver colonoscopy across the three bowel cancer screening programmes in $\mathrm{C} \& \mathrm{M}$. There was expected to be significant delays in the accreditation process for any new endoscopist due to the suspension of training courses and assessment days.
Methods The C \& M Endoscopy Network is made up of eight Trusts on 12 sites and it is led by two Clinical Leads, a Programme Manager and a Project Manager. This Team created a project proposal to deliver Accelerated Accreditation of BCSP Colonoscopists. Funding was obtained from NHSE. The local process was approved by JAG which is the accreditation authority for the bowel cancer screening programme. This accelerated accreditation process included the development and delivery of webinars by our Clinical Lead, local assessment to replace pre accreditation courses and assessors travelling around the network to support candidates. The Mersey School of Endoscopy set up four accreditation assessment days in rapid succession and a new assessment site was added due to the efforts of the local Bowel Cancer Screening Managers. External assessors travelled across the country to support this pilot and candidates were supported by their local clinicians. New assessors were also provided training.

Results 15 candidates were put through the accelerated accreditation process to fill the workforce gap in the three BCSP across Cheshire and Merseyside over a short period of four months compared to the usual prolonged process. The Bowel Cancer screening programmes combined Clinical establishment will increase from 18 to 33 - an increase of $83 \%$. Clinicians will have honorary contracts at every Trust to allow cross trust working so that they are able to maintain the minimal numbers required for accreditation. In addition, this allowed accreditation for four additional assessors and of a new assessment site - both of which will help in future sustainability.

Conclusion This accelerated accreditation can be used a model for other programmes across the country to help with the work force gap.

\section{PTU-85 PLAYING HIDE AND SEEK: QUALITATIVE RESEARCH AND GASTROENTEROLOGY}

${ }^{1,2}$ Mohammad Farhad Peerally*, ${ }^{3}$ Vikram Mohanan, ${ }^{3}$ Ajay Verma, ${ }^{2} J o h n$ DeCaestecker. ${ }^{1}$ SAPPHIRE, Department of Health Sciences, University of Leicester, Leicester, UK; ${ }^{2}$ Digestive Diseases Centre, University Hospitals of Leicester NHS Trust, Leicester, UK; ${ }^{3}$ Digestive Diseases Unit, Kettering General Hospital NHS Foundation Trust, Kettering, UK

\subsection{6/gutjnl-2021-BSG.385}

Introduction Qualitative research includes a range of methodologies seeking to capture views of patients and practitioners on clinical interventions, and their acceptability and effectiveness in the real world. The lack of qualitative research in clinical journals, such as the BMJ, is recognised as a major concern by sections of the research community. ${ }^{1}$ No data is available on the existence of a similar issue in GI research. We seek to determine the number of original qualitative studies (including mixed-methods) published in the top $10 \mathrm{GI}$ journals, ranked according to the SCImago Journal Rank, a measure of the scientific influence of journals accounting for both citations and prestige, and identify whether qualitative research in GI is conducted in the UK, based on UK Clinical Research Network (CRN) data.

Method An advanced Pubmed search was conducted in May 2021, using a validated search strategy including a combination of terms such as 'interview', 'qualitative' and 'experience', 2 to identify qualitative studies published between 2000 2021 in the top 10 GI journals. The UK CRN database was interrogated to identify actively recruiting GI studies which include qualitative methods on the NIHR portfolio. 